\title{
Search for a bound H-dibaryon using local six-quark interpolating operators
}

\author{
Jeremy Green*, Anthony Francis, Parikshit Junnarkar, Chuan Miao, Thomas Rae, \\ and Hartmut Wittig \\ Institut für Kernphysik, Helmholtz-Institut Mainz, and PRISMA Cluster of Excellence, \\ Johannes Gutenberg-Universität Mainz, D-55099 Mainz, Germany \\ E-mail: green@kph.uni-mainz.de, francis@kph.uni-mainz.de, \\ junnarka@kph.uni-mainz.de, chuan@kph.uni-mainz.de, \\ thrae@uni-mainz.de, wittig@kph.uni-mainz.de
}

\begin{abstract}
We present early results from a lattice QCD study seeking a bound $H$-dibaryon using $N_{f}=2$ flavors of $O(a)$ improved Wilson fermions and a quenched strange quark. We compute a matrix of two-point functions using operators consisting of the two independent local products of six positive-parity-projected quarks with the appropriate quantum numbers, which belong to the singlet and 27-plet irreducible representations of flavor SU(3). To expand this basis, we also independently vary the quark-field smearing, and apply a new scheme to reduce the noise caused by smearing. We then find the ground-state mass by solving the generalized eigenvalue problem. We show results from ensembles with pion masses $451 \mathrm{MeV}$ and $1 \mathrm{GeV}$, and compare with other lattice calculations.
\end{abstract}

The 32nd International Symposium on Lattice Field Theory

23-28 June, 2014

Columbia University New York, NY

${ }^{*}$ Speaker. 


\section{Introduction}

The $H$-dibaryon is a conjectured six-quark hadron with quark content $u u d d s s$ that is a scalar and a flavor singlet [1]. Among the experimental constraints on its existence is the "Nagara" event from E373 at KEK [2], which found a ${ }_{\Lambda \Lambda}^{6} \mathrm{He}$ double-hypernucleus with binding energy $B_{\Lambda \Lambda}=$ $6.91 \pm 0.16 \mathrm{MeV}$ [3]. The absence of a strong decay ${ }_{\Lambda \Lambda}^{6} \mathrm{He} \rightarrow{ }^{4} \mathrm{He}+H$ implies

$$
m_{H}>2 m_{\Lambda}-B_{\Lambda \Lambda} .
$$

Lattice QCD studies of a possible $H$-dibaryon have been done for nearly thirty years [4]; however, aside from this work, calculations with dynamical fermions have only been done by two collaborations: NPLQCD $[5,6,7]$ and $\operatorname{HALQCD}[8,9,10]$. These calculations have generally found a bound $H$-dibaryon at fairly heavy quark masses, with a binding energy that becomes smaller when the quark masses are decreased.

\section{Lattice methodology}

For a set of interpolating operators $\mathscr{O}_{i}$, we compute a matrix of zero-momentum two-point functions,

$$
C_{i j}(t)=\sum_{x}\left\langle\mathscr{O}_{i}\left(x, t_{0}+t\right) \mathscr{O}_{j}^{\dagger}\left(x_{0}, t_{0}\right)\right\rangle .
$$

We then find effective masses in two ways: from its diagonal elements,

$$
m_{\mathrm{eff}, i}(t)=\frac{1}{\Delta t} \log \frac{C_{i i}(t)}{C_{i i}(t+\Delta t)},
$$

and from using the variational method [11] by solving the generalized eigenvalue problem (GEVP),

$$
C_{i j}(t+\Delta t) v_{j}(t)=\lambda(t) C_{i j}(t) v_{j}(t) ; \quad m_{\mathrm{eff}}(t)=\frac{-\log \lambda(t)}{\Delta t} .
$$

Both of these effective masses will approach the ground-state mass from above, with exponentiallydecaying excited-state contamination; the variational method serves to eliminate contributions from the lowest-lying excited states [12].

We construct interpolating operators using products of six positive-parity-projected smeared quarks fields, of the form

$$
[a b c d e f]=\varepsilon^{i j k} \varepsilon^{l m n}\left(b_{i}^{T} C \gamma_{5} P_{+} c_{j}\right)\left(e_{l}^{T} C \gamma_{5} P_{+} f_{m}\right)\left(a_{k}^{T} C \gamma_{5} P_{+} d_{n}\right),
$$

where $P_{+}=\left(1+\gamma_{0}\right) / 2$. There are two such local operators that can couple to an $H$-dibaryon in a calculation with degenerate $u$ and $d$ quarks [13, 14]:

$$
\begin{aligned}
H^{\mathbf{1}} & =\frac{1}{48}([\text { sudsud }]-[\text { udusds }]-[\text { dudsus }]) \\
H^{\mathbf{2 7}} & =\frac{1}{48 \sqrt{3}}(3[\text { sudsud }]+[\text { udusds }]+[\text { dudsus }]),
\end{aligned}
$$

which belong to the singlet and 27-plet irreps of flavor SU(3). 
The standard smearing procedure uses Wuppertal smearing [15],

$$
\tilde{q}=(1+\alpha H)^{n} q
$$

where $H$ is the gauge-covariant spatial hopping term constructed using spatially APE-smeared gauge links [16]; generically we write the smearing kernel as $S_{a b}(x, y ; t)$. This tends to introduce noise due to fluctuations in the shape of the smeared field, especially after many steps of smearing [17]. To first approximation, these fluctuations affect the overall amplitude of the smeared quark field and could be reduced by normalizing:

$$
\tilde{q}_{N 1}(x, t)=\frac{1}{N(x, t)} \tilde{q}(x, t), \quad N(x, t)=\sqrt{\sum_{y, a, b}\left|S_{a b}(x, y ; t)\right|^{2}} .
$$

This is simple to apply to a point source but would be rather expensive to compute at the sink. Instead, we introduce a variant of this method, timeslice-normalized smearing, with the normalization factor summed over each timeslice using stochastic estimation:

$$
\tilde{q}_{N}(x, t)=\frac{1}{N(t)} \tilde{q}(x, t), \quad N(t)^{2}=\frac{1}{n_{\text {noise }}} \sum_{x, y, a, b, i}\left|S_{a b}(x, y ; t) \eta_{b}^{(i)}(y, t)\right|^{2} \approx \sum_{x, y, a, b}\left|S_{a b}(x, y ; t)\right|^{2},
$$

where $\eta^{(i)}$ are noise vector fields with expectation value $E\left[\eta^{(i)} \eta^{(j) \dagger}\right]=\delta^{i j} I$. A key feature of this prescription is that a correlator with timeslice-normalized smearing can be obtained from one with ordinary smearing by simple multiplication of the smearing factors: for a dibaryon operator, $C_{N}\left(t_{f}, t_{i}\right)=\left(\frac{1}{N\left(t_{i}\right) N\left(t_{f}\right)}\right)^{6} C\left(t_{f}, t_{i}\right)$, where this is applied individually to each sample ${ }^{1}$. In practice, we used $160 Z_{4}$ noise vectors with color dilution, which has the same computational cost as smearing ten propagators.

To obtain high statistics, we also make use of the all-mode-averaging (AMA) technique [18, 19], computing many samples with lower-precision propagator solves and applying a bias correction using a relatively small number of high-precision solves.

\section{Results}

We performed calculations on two ensembles generated within and provided to us by the CLS effort, with $N_{f}=2$ non-perturbatively clover-improved Wilson fermions, labeled E1 and E5 [20], which have lattice spacing $a=0.063 \mathrm{fm} \mathrm{[21]} \mathrm{and} \mathrm{lattice} \mathrm{volume} 64 \times 32^{3}$. We used a quenched strange quark with hopping parameter $\kappa_{s}$ tuned such that the ratio $\left(m_{K}^{2}-\frac{1}{2} m_{\pi}^{2}\right) / m_{\Omega}^{2}$ takes its physical value. However, on the E1 ensemble we deviated slightly from this tuning to set $\kappa_{s}$ equal to the light-quark hopping parameter in order to have SU(3)-flavor symmetry. The basic information about these two ensembles is summarized in Table 1.

The E5 ensemble has a pion mass of $451 \mathrm{MeV}$. We computed $H$-dibaryon two-point functions using both point and smeared $(n=140, \alpha \approx 0.75)$ quark fields. Together with the singlet and

\footnotetext{
${ }^{1}$ Note that although $E\left[N(t)^{2}\right]=\sum_{x} N(x, t)^{2}$ does not imply $E\left[N(t)^{-6}\right]=\left(\sum_{x} N(x, t)^{2}\right)^{-3}$, with sufficiently many noise sources the difference is small, and its symmetry properties are never spoiled. To be precise: as long as $t_{f} \neq t_{i}$, the expectation value of $C_{N}$ is a two-point function constructed from quark fields $\tilde{q}_{N}(x, t)=E\left[N(t)^{-6}\right]^{1 / 6} \tilde{q}(x, t)$, which depend on the precise details of the set of noise vectors.
} 


\begin{tabular}{l|cc|cc|rrr} 
Label & $\kappa_{u d}$ & $\kappa_{s}$ & $m_{\pi}$ & $m_{\pi} L$ & $N_{\text {conf }}$ & $N_{\text {src }}$ & $N_{\text {samp }}$ \\
\hline E1 & 0.13550 & 0.135500 & $1 \mathrm{GeV}$ & 10 & 168 & 128 & 43008 \\
E5 & 0.13625 & 0.135546 & $451 \mathrm{MeV}$ & 4.6 & 1881 & 16 & 60192
\end{tabular}

Table 1: Ensemble parameters. Note that $N_{\text {src }}$ indicates the number of low-precision source points used per configuration; in both cases one high-precision source was used. We additionally construct operators using $P_{-}=\left(1-\gamma_{0}\right) / 2$ in place of $P_{+}$in Eq. (2.4) to yield both forward and backward-propagating states from each source and thus two samples per source, using time-reversal symmetry.
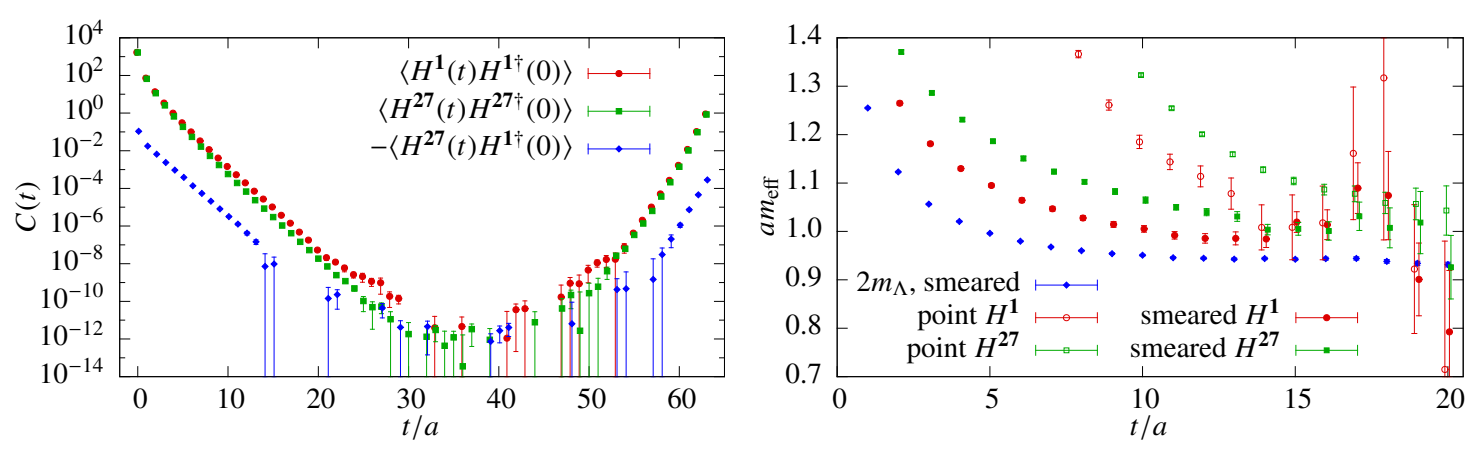

Figure 1: Data from the E5 ensemble. Left: Diagonal and off-diagonal two-point functions of the two smeared operators. Right: Diagonal effective masses of the four operators, along with twice the $\Lambda$ effective mass.

27-plet combinations, this yields four interpolating operators. The two-point functions computed with smeared operators are shown in Fig. 1 (left); we find that the cross-correlator between the singlet and 27-plet operators is suppressed by $2-3$ orders of magnitude, indicating that breaking of flavor-SU(3) is small.

As the lattice spacing is fairly fine, we compute effective masses with a time step $\Delta t=3 a$. For the four diagonal two-point functions, the effective masses are shown in Fig. 1 (right), along with twice the effective mass of the $\Lambda$ baryon. All four $H$-dibaryon effective masses remain clearly above the $2 m_{\Lambda}$ threshold until their signals deteriorate. As should be expected, smearing reduces the coupling to excited states. In addition, the effective masses of the flavor-singlet operators approach the ground state faster than the 27-plet operators. Therefore, we consider the singlet operator with smearing as the "best" single operator; indeed, it is the first to reach a possible plateau at around $t / a=12$. For a final analysis on this ensemble, we use the variational method with the full $4 \times 4$ matrix of two-point functions. The effective mass as determined from the GEVP is shown in Fig. 3 (left), alongside the effective mass from the "best" single operator and twice the $\Lambda$ effective mass. The variational approach yields effective masses that are slightly lower than the "best" operator, and thus a faster approach to a plateau, at the cost of increased statistical uncertainty. Neither approach shows a clear sign of a plateau below the $2 m_{\Lambda}$ threshold and we conclude that the data on this ensemble do not indicate the presence of a bound $H$-dibaryon.

As other calculations have found a more strongly bound state at higher pion masses, we used the E1 ensemble, which has a pion mass of $1 \mathrm{GeV}$, for comparison. Since the light and strange quarks are degenerate, there is no mixing between the singlet and 27-plet operators. Therefore, in order to expand the basis of operators in the singlet channel, we used additional smearings: 

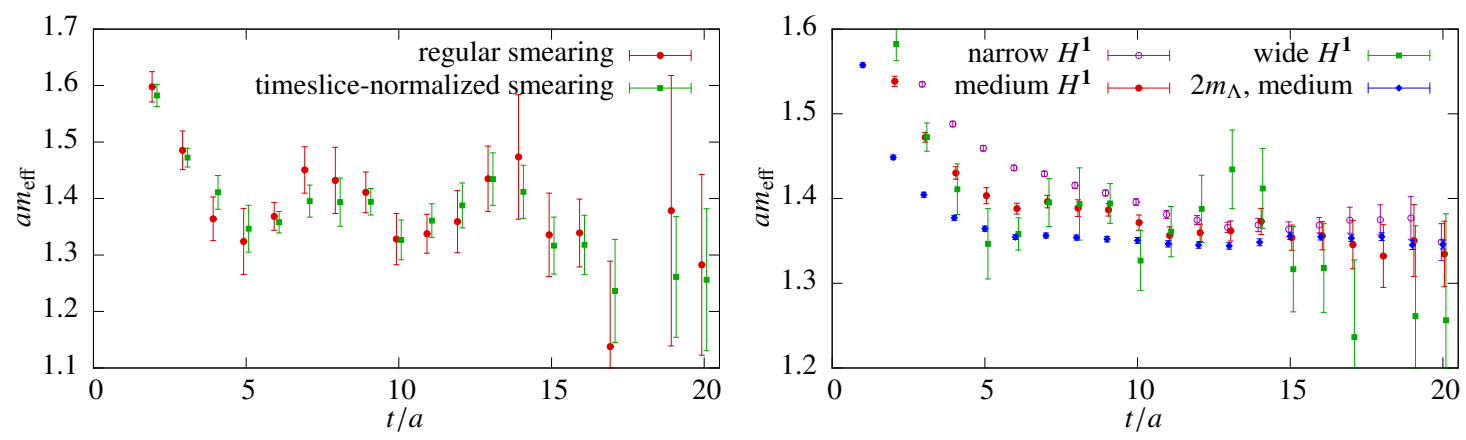

Figure 2: Effective masses from the E1 ensemble. Left: Comparison of regular and timeslice-normalized smearing, for the "wide" singlet operator. Right: Diagonal effective masses of the three operators, along with twice the $\Lambda$ effective mass.
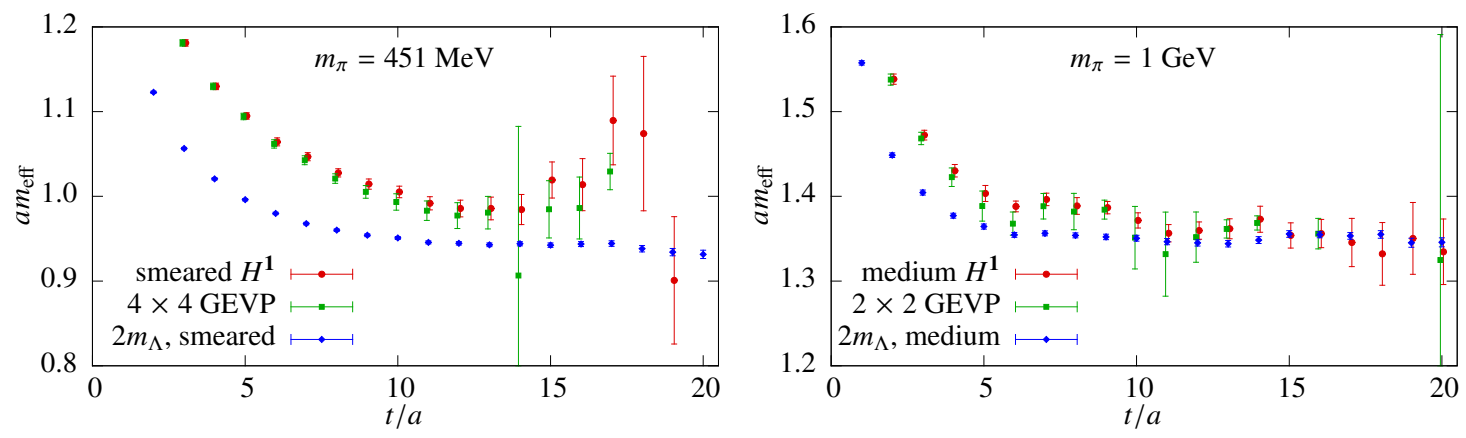

Figure 3: Effective masses on the two ensembles, in each case comparing results from the "best" single operator (see text) and from the variational method against twice the $\Lambda$ effective mass. Left: E5 ensemble. Right: E1 ensemble.

in addition to the same smearing used on E5, which we call "medium", we added "narrow" and "wide" with half and twice as many steps, for a total of three singlet interpolating operators.

The "wide" smearing introduces additional noise, and thus it benefits the most from timeslicenormalized smearing. This is shown in Fig. 2 (left), where a considerable reduction in noise can be seen in this operator's effective mass. The diagonal effective masses for the three singlet operators are shown in Fig. 2 (right). The "wide" operator approaches a plateau at a similar rate as the "medium" operator, although it is much noisier. The "narrow" operator has the least noise of the three, but it has greater contamination from excited states. The plateaus reached by the operators are consistent with the $2 m_{\Lambda}$ threshold; i.e., present statistics are insufficient to determine if the plateaus lie above or below it.

For the variational analysis on E1, we omit the "wide" operator due to its high level of noise and solve the GEVP for the remaining $2 \times 2$ matrix of two-point functions. The resulting effective mass is shown in Fig. 3 (right), alongside the effective mass from the "medium" operator and $2 m_{\mathrm{eff}, \Lambda}$. As was the case for E5, the variational-method result has a slightly faster approach to a plateau, however it is somewhat noisier than the single operator. There is no plateau clearly below the $2 m_{\Lambda}$ threshold, and thus we do not find evidence for a bound $H$-dibaryon on this ensemble; however, present statistics are insufficient to rule out the possibility. 


\section{Discussion and outlook}

The absence of a bound $H$-dibaryon is in contrast with other lattice QCD calculations using dynamical fermions. In particular, NPLQCD, computing the spectrum using two-point functions on the lattice, found a binding energy of $75 \mathrm{MeV}$ using ensembles with three degenerate quark flavors and pion masses of about $800 \mathrm{MeV}$ [7], which corresponds to about 0.024 in lattice units on the "E" lattices. This is clearly inconsistent with the data on E1, except at rather large source-sink separations where the statistical uncertainty is much greater.

The lattice ensembles used in this work and by NPLQCD are somewhat different. In particular, this work lacks a finite-volume study, which may be important for multi-baryon states; the volumes used in Ref. [7] were all larger than the single volume used in this work, however no significant dependence of the $H$-dibaryon binding energy on the lattice volume was found in that work. In addition, this work used a significantly finer lattice spacing and a quenched strange quark; it is possible that these contribute to the difference in results.

A key difference between this work and that of NPLQCD is the interpolating operators: whereas we use a symmetric setup with the same six-quark operators at the source (at a single point) and at the sink (projected to zero momentum), NPLQCD used an asymmetric setup. At the source, they had six-quark operators at a point, and at the sink, they used a product of two individually momentum-projected three-quark operators (i.e., two-baryon operators). It may be the case that the two-baryon operators couple much better to the ground state in this channel, and that very large source-sink separations would be required to obtain the ground-state energy using only six-quark operators.

Another related possibility is that a reduced statistical uncertainty would reveal a plateau below the $2 m_{\Lambda}$ threshold at somewhat larger source-sink separations.

We plan to address both of the latter possibilities by supplementing our set of operators with two-baryon operators at the sink, in addition to increasing statistics. This will allow for a cleaner comparison with NPLQCD, as well as the qualitative information about the ground state that can be obtained by studying its relative couplings to six-quark and two-baryon operators.

\section{Acknowledgments}

We thank our colleagues within CLS for sharing the lattice ensembles used. This work made use of the "Wilson" cluster at the Institute for Nuclear Physics and the "Clover" cluster at the Helmholtz Institute Mainz, both at the University of Mainz; we thank Christian Seiwerth and Dalibor Djukanovic for technical support. We are also grateful for computer time allocated to project HMZ21 on the BG/Q JUQUEEN computer at NIC, Jülich.

\section{References}

[1] R. L. Jaffe, Perhaps a stable dihyperon, Phys. Rev. Lett. 38 (1977) 195-198.

[2] H. Takahashi, J. K. Ahn, H. Akikawa, S. Aoki, K. Arai et al., Observation of a ${ }_{\Lambda \Lambda}{ }^{6} \mathrm{He}$ double hypernucleus, Phys. Rev. Lett. 87 (2001) 212502.

[3] KEK-E176, E373, and J-PARC-E07 Collaboration, K. Nakazawa, Double- $\Lambda$ hypernuclei via the $\Xi^{-}$ hyperon capture at rest reaction in a hybrid emulsion, Nucl. Phys. A 835 (2010) 207-214. 
[4] P. B. Mackenzie and H. B. Thacker, Evidence against a stable dibaryon from lattice QCD, Phys. Rev. Lett. 55 (1985) 2539.

[5] NPLQCD Collaboration, S. R. Beane et al., Evidence for a bound H-dibaryon from lattice QCD, Phys. Rev. Lett. 106 (2011) 162001 [1012.3812].

[6] NPLQCD Collaboration, S. R. Beane, E. Chang, W. Detmold, B. Joó, H. W. Lin et al., Present constraints on the H-dibaryon at the physical point from lattice QCD, Mod. Phys. Lett. A 26 (2011) 2587-2595 [1103.2821].

[7] NPLQCD Collaboration, S. R. Beane, E. Chang, S. D. Cohen, W. Detmold, H. W. Lin et al., Light nuclei and hypernuclei from quantum chromodynamics in the limit of $S U(3)$ flavor symmetry, Phys. Rev. D 87 (2013) 034506 [1206.5219].

[8] HAL QCD Collaboration, T. Inoue et al., Bound H-dibaryon in flavor SU(3) limit of lattice QCD, Phys. Rev. Lett. 106 (2011) 162002 [1012.5928].

[9] HAL QCD Collaboration, T. Inoue et al., Two-baryon potentials and H-dibaryon from 3-flavor lattice QCD simulations, Nucl. Phys. A 881 (2012) 28-43 [1112.5926].

[10] HAL QCD Collaboration, T. Inoue, Study of H-dibaryon mass in lattice QCD, PoS LATTICE2012 (2012) 144 [1212.4230].

[11] M. Lüscher and U. Wolff, How to calculate the elastic scattering matrix in two-dimensional quantum field theories by numerical simulation, Nucl. Phys. B 339 (1990) 222-252.

[12] B. Blossier, M. Della Morte, G. von Hippel, T. Mendes and R. Sommer, On the generalized eigenvalue method for energies and matrix elements in lattice field theory, JHEP 0904 (2009) 094 [0902.1265].

[13] J. F. Donoghue, E. Golowich and B. R. Holstein, Weak decays of the H dibaryon, Phys. Rev. D 34 (1986) 3434.

[14] I. Wetzorke, F. Karsch and E. Laermann, Further evidence for an unstable H dibaryon?, Nucl. Phys. Proc. Suppl. 83 (2000) 218-220 [hep-lat/9909037].

[15] S. Güsken, A Study of smearing techniques for hadron correlation functions, Nucl. Phys. Proc. Suppl. 17 (1990) 361-364.

[16] APE Collaboration, M. Albanese et al., Glueball masses and string tension in lattice QCD, Phys. Lett. B 192 (1987) 163-169.

[17] G. M. von Hippel, B. Jäger, T. D. Rae and H. Wittig, The shape of covariantly smeared sources in lattice QCD, JHEP 1309 (2013) 014 [1306.1440].

[18] T. Blum, T. Izubuchi and E. Shintani, New class of variance-reduction techniques using lattice symmetries, Phys. Rev. D 88 (2013) 094503 [1208. 4349].

[19] E. Shintani, Error reduction with all-mode-averaging in Wilson fermion, POS (LATTICE2014) 124.

[20] P. Fritzsch, F. Knechtli, B. Leder, M. Marinkovic, S. Schaefer et al., The strange quark mass and Lambda parameter of two flavor QCD, Nucl. Phys. B 865 (2012) 397-429 [1205 . 5380 ].

[21] S. Capitani, M. Della Morte, G. von Hippel, B. Knippschild and H. Wittig, Scale setting via the $\Omega$ baryon mass, PoS LATTICE2011 (2011) 145 [1110 . 6365]. 